Research Article

\title{
Effects of Dendrobium Polysaccharides on the Functions of Human Skin Fibroblasts and Expression of Matrix Metalloproteinase-2 under High-Glucose Conditions
}

\author{
Yajia Li $\mathbb{D}^{1},{ }^{1}$ Ziqin Cao $\mathbb{D}^{2},{ }^{2}$ Qiangxiang Li $\mathbb{D}^{3,4,5}$ Chenxu Wang, ${ }^{5}$ and Zhuo Zhou ${ }^{5}$ \\ ${ }^{1}$ Department of Dermatology, Xiangya Hospital, Central South University, Changsha, China \\ ${ }^{2}$ Department of Orthopaedics, The Second Xiangya Hospital, Central South University, Changsha, Hunan, China \\ ${ }^{3}$ National Clinical Research Center for Geriatric Disorders of Xiangya Hospital, \\ Central South University (Sub-Center of Ningxia), Yinchuan, Ningxia Hui Autonomous Region 750001, China \\ ${ }^{4}$ Ningxia Geriatric Disease Clinical Research Center, People's Hospital of Ningxia Hui Autonomous Region, Yinchuan, \\ Ningxia Hui Autonomous Region 750001, China \\ ${ }^{5}$ Hunan People's Hospital, Department of Hunan Institute of Geriatrics, Changsha 410002, China
}

Correspondence should be addressed to Qiangxiang Li; liqiangxiang@nxmu.edu.cn

Received 8 June 2020; Revised 25 January 2021; Accepted 24 February 2021; Published 10 March 2021

Academic Editor: Davide Francomano

Copyright (C) 2021 Yajia Li et al. This is an open access article distributed under the Creative Commons Attribution License, which permits unrestricted use, distribution, and reproduction in any medium, provided the original work is properly cited.

\begin{abstract}
The effects of Dendrobium polysaccharides (PDC) on the functions of human skin fibroblasts (HSFs) and expression of matrix metalloproteinase-2 under high-glucose conditions and exploration of the underlying mechanism remain unclear. We used the 3(4,5-dimethylthiazol-2-yl)-2,5-diphenyltetrazolium bromide (MTT) analysis and flow cytometry to evaluate the cell viability and apoptosis. The collagen levels were determined by the Sircol ${ }^{\mathrm{TM}}$ Collagen Assay. Real-time quantitative polymerase chain reaction (RT-PCR) was used to detect the expression of matrix metalloproteinase-2 (MMP-2) and matrix metalloproteinase inhibitor (TIMP-2) mRNA. We found the following: (1) under the high-glucose condition, the HSF cell viability, the expression of TIMP-2 mRNA, and the collagen levels were reduced, while the apoptosis rate and the expression of MMP-2 mRNA increased $(P<0.05)$. (2) In the high-glucose + PDC group, the PDC reversed the changes in the collagen level, viability, and apoptosis rate of the HSF cells caused by high glucose, with the expression of protein and TIMP-2 mRNA increased and the level of MMP-2 mRNA decreased $(P<0.05)$. This is the first time attempting to reveal that PDC can exhibit protective effects on HSF under high-glucose conditions, which may be related to the upregulation of the TIMP-2 expression and inhibition of the MMP-2 expression.
\end{abstract}

\section{Introduction}

The skin of diabetic patients is known to be susceptible to damage and does not heal easily following an injury. Diabetic skin lesions are related to diabetic vascular disease, neuropathy, cell dysfunction, and abnormal cytokine secretion; however, the specific mechanism for their formation remains unknown $[1,2]$. Thus, evaluation of the molecular mechanism leading to the development of diabetic skin ulcers and the determination of appropriate interventions are of great significance for the diagnosis and effective treatment of this condition. Human skin fibroblasts (HSFs) are a type of repair cells in the dermis and play an important role in moderate tissue metabolism. Cells of this type also secrete collagen as well as noncollagen components, such as the outer matrix, and play a critical role in wound healing $[3,4]$. In addition, they are important in maintaining cell elasticity, cultivating water, and supporting epidermal cells [5-8]. It has been determined that fibroblasts are crucial effector cells in the processes related to diabetic skin lesions and participate in the entire wound repair process. The changes in the biological characteristics of these cells are fundamental to the development of diabetic skin lesions. It is noteworthy that the decrease in the number and activity of fibroblasts is also one of the reasons for the reduced synthesis of collagen $[9,10]$. Many in-depth studies have shown that 
the amount and vitality of skin fibroblasts are also important in diabetic skin injury and healing $[11,12]$. Dendrobium polysaccharide, also called polysaccharides of Dendrobium candidum (PDC), is the main bioactive substance of Dendrobium candidum. Previous studies confirmed that PDC could inhibit islet cell apoptosis and necrosis, protect islet cells, and prevent diabetes. In addition, they have also been shown to prevent calcium overload and inhibit corneal epithelial cell apoptosis. PDC prevent skin photoaging; therefore, they display protection and repair properties [13-15]. However, the effects on human skin fibroblasts under high-glucose conditions are yet to be confirmed. Consequently, in the present study, we used various concentrations of PDC to treat HSF in vitro under high-glucose (HG) conditions. We observed the effects of the PDC on the activity and apoptosis of HSF, as well as on the expression of matrix metalloproteinase-2 (MMP-2). The key aim was to establish the protective properties of the PDC on HSF in diabetes and to provide new directions for the treatment of diabetic skin lesions.

\section{Materials and Methods}

2.1. Experimental Materials. Trypsin, Dulbecco's Modified Eagle's Medium (DMEM) culture solution, and low-glucose DMEM culture medium were obtained from Gibco (USA). Fetal bovine serum was purchased from Gibco (USA) or Hangzhou Sijiqing Biological Engineering Material Co. Ltd. (China). Medium-strength protein lysate, NA-Red, and Ponceau S solution were purchased from Biyuntian Biotechnology (China). Biocolor Sircol Soluble Collagen Assay was purchased from Biocolor (UK). The polysaccharide of Dendrobium candidum (PDC) was prepared in house. The $\mathrm{CO}_{2}$ incubator was obtained from Wuhan Huahai or Olympus (China). The KH-600DB data ultrasonic cleaner was purchased from JBtek (USA), while the BD FACSCalibur flow cytometer was obtained from Becton Dickinson (USA).

\subsection{Extraction of Polysaccharide of Dendrobium candidum.} The extraction process of polysaccharides from the protocorm of Dendrobium officinale has been studied. The process in our study is as follows: raw material, weighing, hot water extraction, rough filtration, evaporation concentration, filtrate standing, vacuum filtration, water bath concentration, freeze-drying, and crude polysaccharide. $2000 \mathrm{~g}$ fresh raw materials of Dendrobium officinale were weighed and cut into small sections to facilitate the extraction of polysaccharides. $200 \mathrm{~g}$ of them were reserved for freeze-drying. The remaining parts were extracted by hot water several times. According to the ratio of medicine and water, the medicine was extracted with hot water (medicine $:$ hot water $=1: 4$ ) for 2 hours, and then the initial extraction solution is reserved, then the mixture was extracted (initial extraction solution: water $=1: 2$ ) for next one hour. Then, the filtrate was evaporated and condensed to a certain volume, and then it was freeze-dried for 24 hours to obtain the crude polysaccharide. The extraction technology of polysaccharides from the protocorm of Dendrobium officinale was optimized. The extraction conditions were established by orthogonal design L (934). The experiment was repeated three times. The crude polysaccharide from the protocorm of Dendrobium officinale was extracted by optimized technology, and the purity was $51.3 \%$. In the experiment, the crude polysaccharide of the Dendrobium candidum protocorm can be dissolved in three distilled water to prepare the initial solution of $10 \mathrm{mg} /$ $\mathrm{ml}$, which is filtered and sterilized. Before use, the medium is added and diluted to the required concentration.

2.3. Primary Culture of HSF. The procedure was conducted based on the previously reported method [16]. The infant foreskin was aseptically removed and treated with penicillin $100 \mu \mathrm{g} / \mathrm{mL}$ and streptomycin $100 \mu \mathrm{g} / \mathrm{mL}$. It was then thoroughly washed with phosphate-buffered saline (PBS) and D-Hanks' solution. After removing the subcutaneous tissue, the skin specimen was cut into small pieces using sterile ophthalmic scissors and placed in a type II collagenase digestion solution at $4^{\circ} \mathrm{C}$ for overnight digestion. The epidermis and dermis were aseptically separated. The epidermis was discarded, whereas the dermis was cut and transferred to a solution containing $0.25 \%$ trypsin (excluding ethylenediaminetetraacetic acid (EDTA)) for approximately $15 \mathrm{~min}$. The digestion was stopped by the addition of a highglucose DMEM medium containing $10 \%$ calf serum. The solution was centrifuged at $800 \mathrm{~g}$ for $5 \mathrm{~min}$, and the resulting pellet was inoculated into a $25 \mathrm{~mL}$ plastic culture flask. Approximately $4 \mathrm{~mL}$ of high-glucose DMEM culture solution containing $10 \%$ calf serum was carefully added as not to float the tissue. The culture flask was placed in a $37^{\circ} \mathrm{C}, 5 \%$ $\mathrm{CO}_{2}$ incubator overnight [16]. The following day, $5 \mathrm{~mL}$ of the medium was added. The primary cells were passaged when they were $>80 \%$ full. During the passage, the cells were digested with $0.25 \%$ trypsin (excluding EDTA). The culture flask was patted during the retraction of the cell body under an inverted microscope before the addition of $10 \%$ calf serum. The digestion was stopped by the addition of the high-glucose DMEM medium. The cells on the wall of the flask were gently pipetted and collected into a $10 \mathrm{~mL}$ centrifuge tube. The cells were centrifuged at $800 \mathrm{~g}$ for $5 \mathrm{~min}$, and the supernatant was discarded. The cells were inoculated and routinely cultured.

2.4. High-Glucose Model Establishment and Experimental Grouping. The HSF cells were cultured and passaged. When the cells reached $80 \%$ confluence, the serum-containing culture medium was discarded. The serum-free medium was changed after $24 \mathrm{~h}$, and cultures containing different concentrations of glucose were subsequently prepared $(5,10,15$, $20,25,30,35$, and $40 \mathrm{mmol} / \mathrm{L}$ of glucose). After observation at $12,24,36,48$, and $72 \mathrm{~h}$, the optimal glucose concentration for the high-glucose model of the HSF cells was established at $25 \mathrm{mmol} / \mathrm{L}$. The experiment was divided into the following groups: (1) control group (control, C): HSF cells were cultured with the DMEM medium containing 10\% fetal bovine serum for $48 \mathrm{~h}$; (2) high-glucose group (high glucose, HG): HSF treated with the culture solution containing 
$25 \mathrm{mmol} / \mathrm{L}$ of glucose for $48 \mathrm{~h}$; (3-5) PDC at different doses: HSF treated with the culture solution containing the 100 , 200 , and $400 \mu \mathrm{g} / \mathrm{mL}$ of PDC for $48 \mathrm{~h}$; and (6-8) high glucose + different doses of the PDC group (HG + PDC): HSF treated with the culture solution containing $25 \mathrm{mmol} / \mathrm{L}$ of glucose and 100, 200, and $400 \mu \mathrm{g} / \mathrm{mL}$ of PDC for $48 \mathrm{~h}$. High mannitol $(25 \mathrm{mmol} / \mathrm{L})$ was utilized as the osmotic pressure control.

2.5. MTT Cell Viability Test. The 3-(4,5-Dimethylthiazol-2yl)-2,5-diphenyltetrazolium bromide (MTT) method was used to detect cell viability. The principle of this assay is that the dehydrogenase enzymes in living cells reduce tetrazolium to a water-insoluble blue product, i.e., formazan, which precipitates in the cells. Conversely, dead cells do not exhibit this property. Dimethyl alum dissolves the blue-purple crystals in the cells, and the color depth is proportional to the amount of formazan $[17,18]$.

2.6. Collagen Detection Method. The cell supernatants of each group were collected for the determination of collagen content in the cell culture medium. $100 \mu \mathrm{L}$ of the culture medium containing collagen was separated, and the concentrated reagents were added. The supernatant was discarded following centrifugation at $4^{\circ} \mathrm{C}$ overnight. Standard collagen was diluted to $0,0.01,0.05,0.1,0.2$, and $1.00 \mathrm{mg} / \mathrm{L}$, respectively. $500 \mu \mathrm{L}$ of Sircol dye was added to each sample and incubated for $30 \mathrm{~min}$. The samples were then washed with Acid-Salt Wash Reagent (R). $250 \mu \mathrm{L}$ of alkaline metal reagent was added to each tube before mixing by the vortex. $200 \mu \mathrm{L}$ of the sample was transferred onto a 96-well plate, which was analyzed by detection utilizing a microplate reader. The detection wavelength was $555 \mathrm{~nm}$, and the absorbance value was adjusted to zero with water. The absorbances of blank reagents, standard collagen, and test samples were measured three times for accuracy. The test error was $\pm 10 \%$.

2.7. Detection of the Effect of PDC on the Apoptosis of HSF in the HG Environment Using Annexin V-FITC/PI and Flow Cytometry. The cells of the nine groups were separated during the logarithmic growth period. Following digestion by pancreatin without EDTA, the cells were placed in centrifuge tubes and rinsed twice with PBS. The cells were subsequently centrifuged for $5 \mathrm{~min}$ at $800 \mathrm{~g}$. The cells were collected and placed in $1 \mathrm{~mL}$ Eppendorf tubes. $500 \mathrm{~mL}$ of binding buffer was added to suspend the cells. $5 \mathrm{~mL}$ of annexin V-FITC and $5 \mathrm{~mL}$ of propidium iodide were added and allowed to react for 5-15 $\mathrm{min}$ at room temperature.

2.8. Determination of the Effect of PDC on the Expression of TIMP-2 $m R N A$ and MMP-2 $m R N A$ in the HSF Cells in the $H G$ Environment Using $R T-q P C R$. Nine groups were treated according to the described procedures. The cells were collected to determine the expression of TIMP-2 mRNA and MMP-2 mRNA in the HSF cells using the real-time quantitative polymerase chain reaction (RT-qPCR). The extraction of the total RNA from the HSF cells, as well as the reverse transcription of RNA and RT-qPCR, was carried out utilizing the SYBR ${ }^{\circledR}$ method. The target gene sequence was searched in the National Center for Biotechnology Information (NCBI). Primer 5 software was used to design the primer. System composition and amplification of quantitative PCR were conducted according to the method previously described by Megha [19] (Table 1).

2.9. Statistical Treatment. All the data were analyzed using the SPSS version 19.0 software package. The measurement data were presented according to the differences between the average \pm standard values. The $t$-test of the individual samples was performed to compare the average values between two groups satisfying the normal distribution and homogeneity of variance. Significance between groups was also evaluated by one-way analysis of variance (ANOVA) followed by a Tukey HSD post hoc test. The single-factor analysis of variance was used for the comparison of average values among the various groups. Findings were given as mean \pm SD and compared by Dunnett's test. All groups were compared in pairs. $P<0.05$ indicated that the difference was of statistical significance.

\section{Results}

3.1. Effects of High Glucose Concentration and PDC on the Viability of the HSF Cells. The obtained results are demonstrated in Figure 1 and Table S1. As can be seen, compared with the control group, the HSF cell viability of the highglucose group was significantly reduced $(P<0.05)$. On the contrary, the cell concentration of the analogous glucose content group was not significantly different from the control group $(P>0.05)$, indicating that high glucose concentration leads to reduced viability of the HSF cells. Moreover, this outcome also suggests that induced cell damage is not related to the osmotic pressure. No significant difference in the cell viability between the Dendrobium polysaccharide $(100,200$, and $400 \mu \mathrm{g} / \mathrm{mL})$ and the control groups was observed $(P>0.05)$. Compared with the highglucose group, the cell viability of the high-glucose + PDC $(100,200$, and $400 \mu \mathrm{g} / \mathrm{mL})$ groups significantly increased, demonstrating a concentration dependence $(P<0.05)$.

3.2. Effects of High Glucose Concentration and PDC on HSF Cell Apoptosis. The results of the analysis are shown in Figure 2 and Table S2. It was determined that the apoptosis rate of the HSF cells in the high-glucose group significantly increased compared with the control group $(P<0.05)$. Conversely, the apoptosis rate of the same mannitol content group was not significantly different from the control group $(P>0.05)$, indicating that high glucose concentration leads to increased apoptosis of the HSF cells. Furthermore, the induced cell damage is not related to the osmotic pressure. Apoptosis rates of the PDC $(100,200$, and $400 \mu \mathrm{g} / \mathrm{mL})$ groups were not significantly different from those of the control group $(P>0.05)$. Notably, compared with the high-glucose group, the high-glucose + PDC (100, 
TABLE 1: Specific primer sequences for RT-qPCR.

\begin{tabular}{lccc}
\hline Gene & & Sequence $\left(5^{\prime}-3^{\prime}\right)$ & $\begin{array}{c}\text { Product } \\
\text { length }(\mathrm{bp})\end{array}$ \\
\hline TIMP-2 & $\begin{array}{c}\text { Forward } \\
\text { Reverse }\end{array}$ & $\begin{array}{c}\text { CCTCTGGCATCTCTTGT } \\
\text { ACCCCACAGCCAGCACTAT }\end{array}$ & 423 \\
\hline Bcl-2 & Forward & $\begin{array}{c}\text { TTCTGGGCAACA } \\
\text { AGTATGA }\end{array}$ & 436 \\
\hline GAPDH & $\begin{array}{c}\text { Forward } \\
\text { Reverse }\end{array}$ & $\begin{array}{c}\text { TCGGACGCCTGGTTAC } \\
\text { CGCT CCTGGA AGATGG }\end{array}$ & 199 \\
\hline
\end{tabular}

Primer design: the target gene sequence was searched in the NCBI. Primer 5 software was used to design the primer.

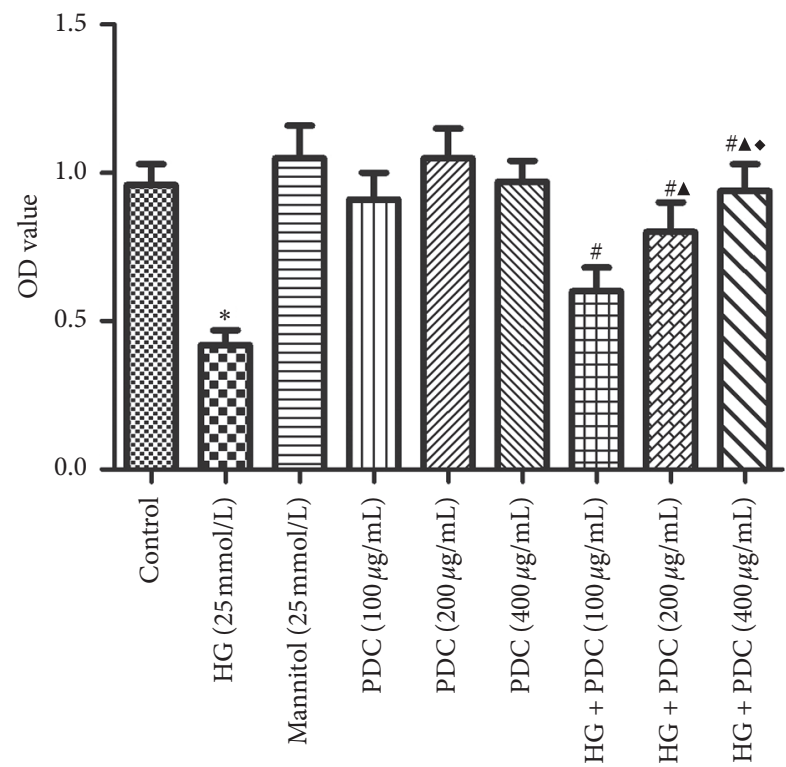

FIGURE 1: Effects of high glucose concentration and PDC on the viability of the HSF cells. HSF cells were incubated at high glucose concentration $(25 \mathrm{mmol} / \mathrm{L})$ or PDC $(100,200$, and $400 \mu \mathrm{g} / \mathrm{mL})$ for $48 \mathrm{~h}$. The MTT assay was used to detect the cell viability. All the data were expressed as $\bar{x} \pm S(n=3) .{ }^{*} P<0.05$, compared with the control group; ${ }^{\#} P<0.05$, compared with the high-glucose group; $\Delta P<0.05$, compared with the high-glucose + Dendrobium polysaccharide $(100 \mu \mathrm{g} / \mathrm{mL})$ group; $\bullet P<0.05$, compared with the highglucose + Dendrobium polysaccharide $(200 \mu \mathrm{g} / \mathrm{mL})$ group.

200 , and $400 \mu \mathrm{g} / \mathrm{mL}$ ) group had reduced high glucose-induced apoptosis in a concentration-dependent manner $(P<0.05)$.

\subsection{Effects of High Glucose Concentration and PDC on HSF} Cell Collagen Content in the Cell Culture Fluid. Compared with the control group, the collagen level in the HSF cell culture fluid of the high-glucose group significantly decreased $(P<0.05)$ (Figure 3 and Table S3). Collagen levels in the cell culture fluid of the PDC $(100,200$, and $400 \mu \mathrm{g} / \mathrm{mL})$ groups did not change compared with the control group $(P>0.05)$. Moreover, in comparison with the high-glucose group, the collagen level in the culture fluid in the highglucose + PDC $(100,200$, and $400 \mu \mathrm{g} / \mathrm{mL})$ groups significantly increased in a concentration-dependent manner $(P<0.05)$.

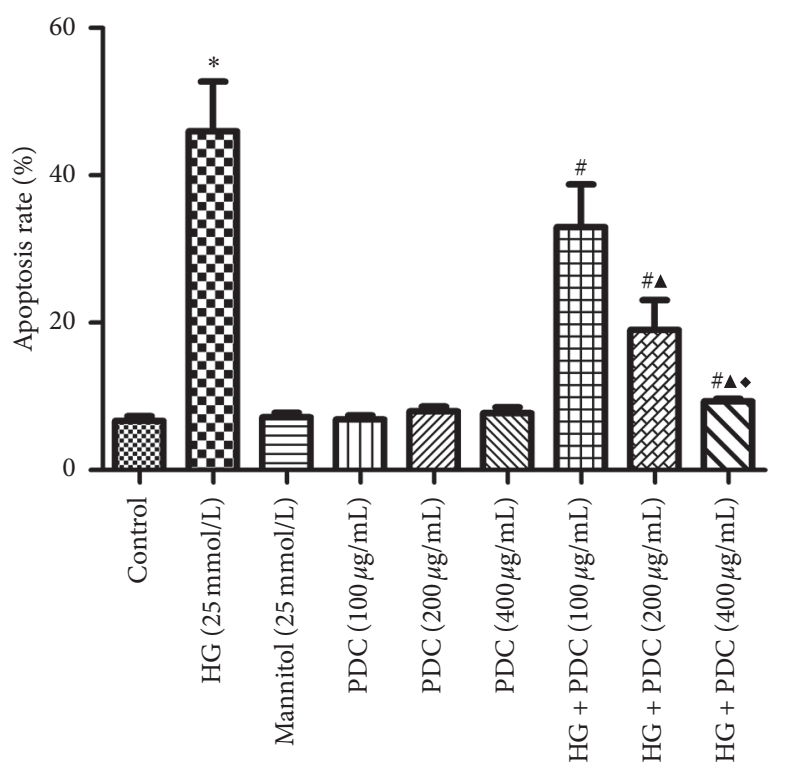

FIGURE 2: Effects of high glucose and PDC on apoptosis of HSF cells. HSF cells were incubated at high glucose concentration $(25 \mathrm{mmol} / \mathrm{L})$ or PDC $(100,200$, and $400 \mu \mathrm{g} / \mathrm{mL})$ for $48 \mathrm{~h}$. Apoptosis was detected by flow cytometry. All the data were expressed as $\bar{x} \pm S \quad(n=3) .{ }^{*} P<0.05$, compared with the control group; ${ }^{\#} P<0.05$, compared with the high-glucose group; ${ }^{\wedge} P<0.05$, compared with the high-glucose + Dendrobium polysaccharide $(100 \mu \mathrm{g} / \mathrm{mL})$ group; $P<0.05$, compared with the highglucose + Dendrobium polysaccharide $(200 \mu \mathrm{g} / \mathrm{mL})$ group.

3.4. Effect of High Glucose Concentration and PDC on the mRNA Expression of MMP-2 and TIMP-2 in the HSF Cells. Compared with the control group, the expression of MMP-2 mRNA significantly increased in the high-glucose HSF cells $(P<0.05)$, while the expression of TIMP-2 mRNA decreased (Figures 4 and 5 and Tables S4 and S5). Compared with the control group, the PDC $(100,200$, and $400 \mu \mathrm{g} / \mathrm{mL})$ downregulated the expression of MMP-2 mRNA and upregulated the expression of TIMP-2 mRNA in the HSF cells in a concentration-dependent manner $(P<0.05)$. Compared with the high-glucose group, the expression of MMP-2 mRNA in the HSF cells in the high-glucose + PDC $(100,200$, and $400 \mu \mathrm{g} / \mathrm{mL}$ ) group was notably reduced, whereas the expression of TIMP-2 mRNA increased in a concentrationdependent manner $(P<0.05)$.

\section{Discussion}

The high glucose environment mimicing diabetic state can not only reduce the ability to migrate and proliferate of fibroblasts, but also increase the cell apoptosis. As previously mentioned, fibroblasts constitute the principal repair cells in wound healing and are some of the main components of the granulation tissue. They synthesize and secrete the extracellular matrix, including collagen, fibronectin, and hyaluronic acid [20-23]. Thus, in the pathological state of diabetes, increased fibroblast apoptosis inevitably hinders the healing of skin ulcers. The dynamic balance of the matrix metalloproteinase (MMP) family is abnormal during diabetes. The increase in the expression of MMPs and enhanced 


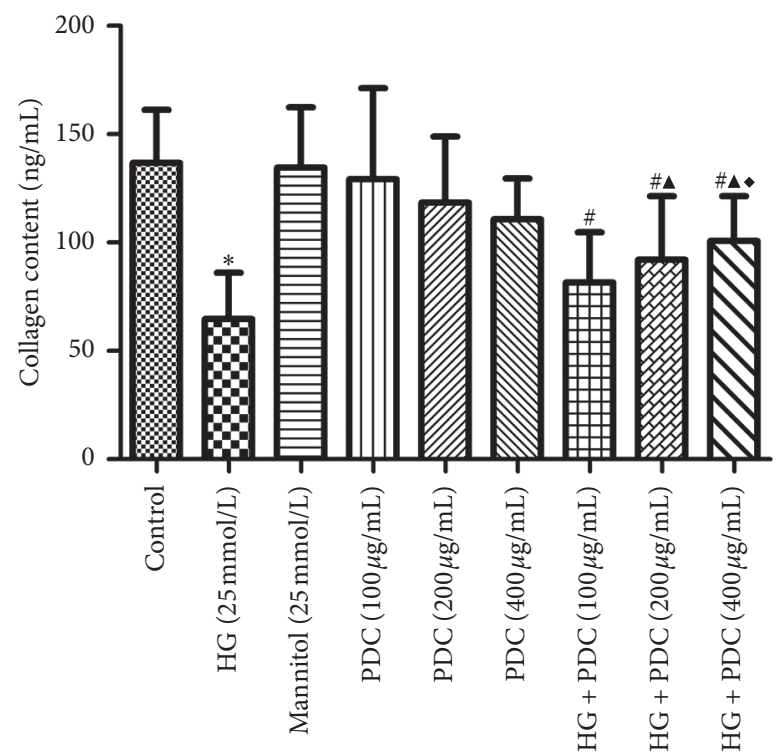

FIGURE 3: Effect of high glucose concentration and PDC on the collagen content in the cell culture fluid. HSF cells were incubated at high glucose concentration $(25 \mathrm{mmol} / \mathrm{L})$ or PDC $(100,200$, and $400 \mu \mathrm{g} / \mathrm{mL})$ for $48 \mathrm{~h}$. The cell culture fluid was collected, and the level of collagen in the culture fluid was detected by a kit method. All the data were expressed as $\bar{x} \pm S(n=3) .{ }^{*} P<0.05$, compared with the control group; ${ }^{\#} P<0.05$, compared with the high-glucose group; ${ }^{\wedge} P<0.05$, compared with the high-glucose + Dendrobium polysaccharide $(100 \mu \mathrm{g} / \mathrm{mL})$ group; $\bullet P<0.05$, compared with the high-glucose + Dendrobium polysaccharide $(200 \mu \mathrm{g} / \mathrm{mL})$ group.

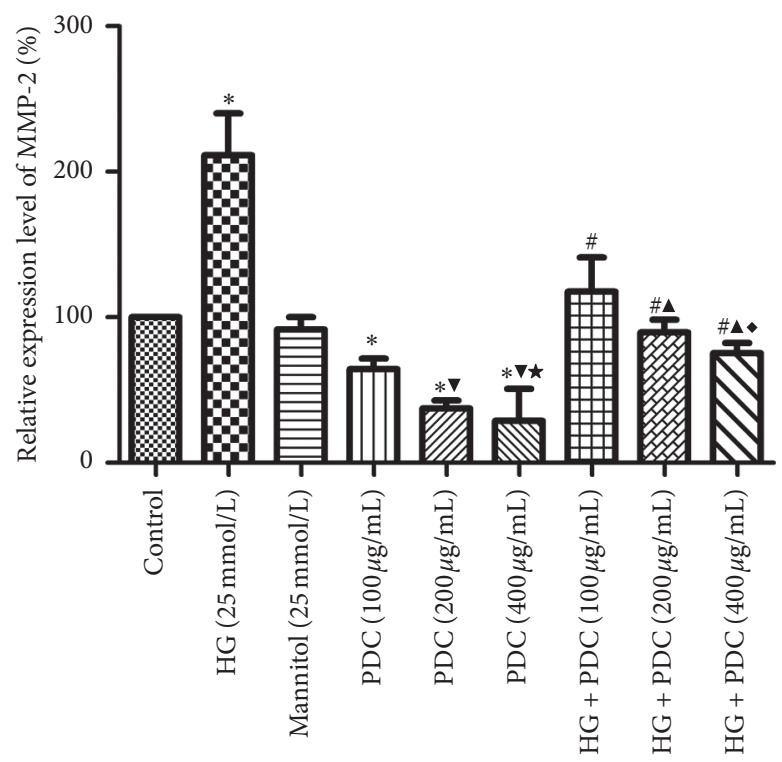

Figure 4: Effects of high glucose concentration and PDC on the mRNA expression of MMP-2 in HSF cells. HSF cells were incubated at high glucose $(25 \mathrm{mmol} / \mathrm{L})$ or PDC $(100,200$, and $400 \mu \mathrm{g} / \mathrm{mL})$ for $48 \mathrm{~h}$. The total RNA of the cells was collected, and the mRNA expression of MMP-2 in the cells was detected by real-time quantitative PCR. All the data were expressed as $\bar{x} \pm S(n=3) .{ }^{*} P<0.05$, compared with the control group; $\nabla^{\nabla}<0.05$, compared with the Dendrobium polysaccharide $(100 \mu \mathrm{g} / \mathrm{mL})$ group; ${ }^{\star} P<0.05$, compared with the Dendrobium polysaccharide $(200 \mu \mathrm{g} / \mathrm{mL})$ group; ${ }^{\#} P<0.05$, compared with the high-glucose group; $P<0.05$, compared with the highglucose + Dendrobium polysaccharide $(100 \mu \mathrm{g} / \mathrm{mL})$ group; ${ }^{\bullet} P<0.05$, compared with the high-glucose + Dendrobium polysaccharide $(200 \mu \mathrm{g} / \mathrm{mL})$ group.

enzyme activity can lead to excessive degradation of the extracellular matrix, resulting in the formation of the chronic refractory wound surface. Studies have shown that MMP-2 is increased in undamaged dermal fibroblasts [24-26].
It has been demonstrated previously that, in traditional Chinese medicine, Dendrobium officinale could strengthen the spleen as well as nourishes the stomach, lungs, and kidneys. And it is typically used to treat chronic gastritis, hypertension, diabetes, and chronic nephritis, as well as 


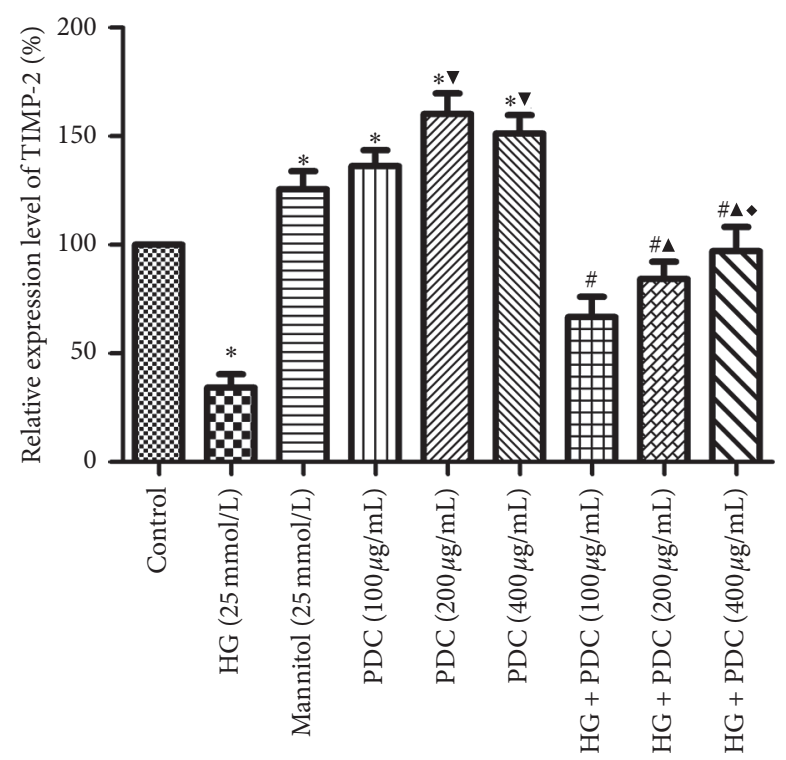

FIGURE 5: Effects of high glucose concentration and PDC on the mRNA expression of TIMP-2 in the HSF cells. HSF cells were incubated at high glucose concentration $(25 \mathrm{mmol} / \mathrm{L})$ or PDC $(100,200$, and $400 \mu \mathrm{g} / \mathrm{mL})$ for $48 \mathrm{~h}$. The total RNA of the cells was collected, and the mRNA expression of TIMP-2 in the cells was detected by real-time quantitative PCR. All the data were expressed as $\bar{x} \pm S(n=3)$. ${ }^{*} P<0.05$, compared with the control group; ${ }^{\nabla} P<0.05$, compared with the Dendrobium polysaccharide $(100 \mu \mathrm{g} / \mathrm{mL})$ group; ${ }^{\#} P<0.05$, compared with the high-glucose group; $\wedge<0.05$, compared with the high-glucose + Dendrobium polysaccharide $(100 \mu \mathrm{g} / \mathrm{mL})$ group; $-P<0.05$, compared with the high-glucose + Dendrobium polysaccharide $(200 \mu \mathrm{g} / \mathrm{mL})$ group.

nephropathy, and is often employed as an antitumor and antiaging agent $[27,28]$. Similar substances were shown to have effects on keratinocytes or endothelial cells, which were also involved in diabetic wound healing. Mo et al. explored the effect of erianin, a bibenzyl compound extracted from Dendrobium chrysotoxum, on proliferation and apoptosis in $\mathrm{HaCaT}$ cells and demonstrated that erianin could be recognized as a potential antipsoriasis drug that inhibited proliferation and induced apoptosis of $\mathrm{HaCaT}$ cells through ROS-mediated JNK/c-Jun and AKT/ mTOR signaling pathways [29]. Erianin was also found to induce a JNK/SAPK-dependent metabolic inhibition in human umbilical vein endothelial cells [30]. The ethanol extract of Dendrobium chrysotoxum Lindl ameliorates retinal angiogenesis during the development of diabetic retinopathy via inhibiting the expression of VEGF/ VEGFR2 and other proangiogenic factors such as MMP-2/ 9 [31]. The present study found that, under high-glucose conditions, the HSF cell viability was significantly reduced, apoptosis rates considerably increased, and collagen levels were notably reduced. Furthermore, the mRNA expression of TIMP-2 decreased, while the mRNA expression of MMP-2 increased in the HSF cells. The addition of PDC $(100,200$, and $400 \mu \mathrm{g} / \mathrm{mL})$ in HSF cells did not have a significant effect on cell viability, apoptosis, or collagen levels. However, PDC $(100,200$, and $400 \mu \mathrm{g} / \mathrm{mL})$ could reverse the changes in the collagen level, viability, and apoptosis rate of the HSF cells caused by high glucose, with the expression of protein and TIMP-2 mRNA increased and the level of MMP-2 mRNA decreased in a concentration-dependent manner $(P<0.05)$. Hence, the present study confirms that PDC can exhibit certain protective effects on the human skin fibroblast function under highglucose conditions. Besides, we inferred that PDC could increase the collagen synthesis of the skin fibroblasts by upregulating TIMP-2 and inhibiting the mRNA expression of MMP-2, which may be conducive to the repair of diabetic skin lesions. Further research is needed to determine the signaling pathways involved in the regulation of collagen synthesis by TIMP-2 in skin fibroblasts. The overexpression of MMP-2 in the involved psoriatic epidermis was found to be accompanied by basement membrane alterations with degraded collagen type IV [32]. Previous studies have also shown that whole-brain irradiation mediates degradation of collagen type IV by altering the balance of MMP-2 and TIMP-2 levels in the brain [33], MMPs and TIMPs are responsible for remodeling in the healthy extracellular matrix, where they are produced in a coordinated manner, and Kozaci et al. found that proMMP-2 levels negatively correlated with the collagen content in herniated disc material [34].

The molecular mechanisms in the pathways regulating TIMP-2 and MMP-2 expressions have been introduced in many diseases, especially diabetes, or different diseases other than diabetes. The study conducted by Ho et al. [35] found that oxidative stress induced by high glucose might be involved in the opposite effects on MMP-2 activation and TIMP-2 downregulation. This reactive oxygen species(ROS-) dependent MMP-2 activation, in turn, mediated high-glucose-induced cell apoptosis in human umbilical vein endothelial cells (HUVECs). Besides, the transforming growth factor (TGF)/SMAD family member 3 (Smad3) pathway was found to regulate MMP/TIMP activity, inducing activation of the fibrosis mediators and suppressing 
the degradation of the extracellular matrix [36-38]. TIMPs could control the MMP activity, and MMP-2 could digest fibrillar collagen peptides and newly formed collagen fibers to degrade collagen $[39,40]$. Furthermore, more evidence showed that TIMP-2 and MMP-2 expressions could play an important role in the development of other diseases. MMP2, TIMP-2, and MMP-2/TIMP-2 ratios may act as biomarkers for susceptibility to systemic lupus erythematosus (SLE) [41]. Pathogenesis of chronic rhinosinusitis might also be related to the regulation of MMP-2 and TIMP-2 expressions. Steroids could inhibit smoke-regulated MMP-2 and TIMP-2 production and activation through the reactive oxygen species (ROS)/PI3K, Akt, and NF- $\kappa \mathrm{B}$ signaling pathways in nasal fibroblasts [42]. However, the causal relationship between MMP-2/TIMP-2 activity and collagen in skin fibroblasts remains unclear and needs to be further investigated. Selective MMP-2 inhibitors and MMP-2 knockout mice could be employed as in-depth pharmacological and genetic approaches to elucidate a mechanistic link among the MMP-2 expression, TIMP-2 expression, and changes in the collagen content in the skin fibroblasts exposed to elevated glucose.

In conclusion, our study revealed that PDC can exhibit protective effects on HSF under high-glucose conditions, which may be related to the upregulation of the TIMP-2 expression and inhibition of the MMP-2 expression, which provided new concepts for the prevention and treatment of diabetic skin ulcers or wounds using traditional Chinese medicine, including Dendrobium officinale.

\section{Data Availability}

The data generated or analyzed during this study are included in this published article. The datasets generated and/ or analyzed during the current study are available from the corresponding author upon reasonable request.

\section{Conflicts of Interest}

The authors declare that they have no conflicts of interest.

\section{Authors' Contributions}

All authors participated in the experiments. Q. L. and Y. L. conceived the experiments. Y. L., Z. C., and C. W. conducted the experiments and drafted the manuscript. Y. L., Z. C., and Z. Z. analyzed the results. All authors reviewed the manuscript, and Q. L. critically reviewed and revised the manuscript. All authors gave final approval to the version submitted for publication.

\section{Acknowledgments}

The authors thank the grants from the National Science Foundation of China (81170823), Hunan Province Philosophy and Social Science Foundation Project of 2018 (18YBA325), Project of Hunan Provincial Department of Education in 2018 (160546), Hunan Provincial Health and Health Commission Scientific Research Fund Project
(C2019055), Hunan Provincial Department of Finance 2017 Project (Xiangcai Finance Agency [2017] 61), Ningxia Hui Autonomous Region Philosophy and Social Science Planning Year Project (19NXAGL01), and Hunan Province's "225" Project Training Discipline Leader Project for HighLevel Health Professionals. This work was supported by Hunan Province Technology Innovation Guidance Program Clinical Medical Technology Innovation Guidance Project (2017SK50510, no. 97).

\section{Supplementary Materials}

Table S1: effects of high glucose concentration and PDC on the viability of the HSF cells. Table S2: effects of high glucose and PDC on apoptosis of HSF cells. Table S3: effect of high glucose concentration and PDC on the collagen content in the cell culture fluid. Table S4: effects of high glucose concentration and PDC on the mRNA expression of MMP-2 in HSF cells. Table S5: effects of high glucose concentration and PDC on the mRNA expression of TIMP-2 in the HSF cells. (Supplementary Materials)

\section{References}

[1] A. L. Lima, T. Illing, S. Schliemann, and P. Elsner, "Cutaneous manifestations of diabetes mellitus: a review," American Journal of Clinical Dermatology, vol. 18, no. 4, pp. 541-553, 2017.

[2] G. Romano, G. Moretti, A. Di Benedetto et al., "Skin lesions in diabetes mellitus: prevalence and clinical correlations," Diabetes Research and Clinical Practice, vol. 39, no. 2, pp. 101-106, 1998.

[3] D. T. Woodley, "Distinct fibroblasts in the papillary and reticular dermis," Dermatologic Clinics, vol. 35, no. 1, pp. 95-100, 2017.

[4] V. Thulabandu, D. Chen, and R. P. Atit, "Dermal fibroblast in cutaneous development and healing," Wiley Interdisciplinary Reviews-Developmental Biology, vol. 7, no. 2, 2018.

[5] F. Quondamatteo, "Skin and diabetes mellitus: What do we know?" Cell and Tissue Research, vol. 355, no. 1, pp. 1-21, 2014.

[6] A. Gomes, C. Teixeira, R. Ferraz, C. Prudêncio, and P. Gomes, "Wound-healing peptides for treatment of chronic diabetic foot ulcers and other infected skin injuries," Molecules, vol. 22, no. 10, p. 1743, 2017.

[7] M. Wolak, T. Staszewska, M. Juszczak, M. Gałdyszyńska, and E. Bojanowska, "Anti-inflammatory and pro-healing impacts of exendin-4 treatment in Zucker diabetic rats: effects on skin wound fibroblasts," European Journal of Pharmacology, vol. 842, pp. 262-269, 2019.

[8] Y. Peng, Y. Zhao, Y. Xie, and X. Y. Lin, "Effects of allogeneic skin fibroblasts on promoting wound healing of diabetic mice and the mechanism," Zhonghua Shao Shang Za Zhi=Zhonghua Shaoshang Zazhi= Chinese Journal of Burns, vol. 34, no. 8, pp. 532-541, 2018.

[9] S. Tajima and S. R. Pinnell, "Collagen synthesis by human skin fibroblasts in culture: studies of fibroblasts explanted from papillary and reticular dermis," Journal of Investigative Dermatology, vol. 77, no. 5, pp. 410-412, 1981.

[10] M. A. Karsdal, S. H. Nielsen, D. J. Leeming et al., "The good and the bad collagens of fibrosis-their role in signaling and organ function," Advanced Drug Delivery Reviews, vol. 121, pp. 43-56, 2017. 
[11] R. R. Driskell and F. M. Watt, "Understanding fibroblast heterogeneity in the skin," Trends in Cell Biology, vol. 25, no. 2, pp. 92-99, 2015.

[12] P. E. Martin, E. M. O'Shaughnessy, C. S. Wright, and A. Graham, "The potential of human induced pluripotent stem cells for modelling diabetic wound healing in vitro," Clinical Science, vol. 132, no. 15, pp. 1629-1643, 2018.

[13] Q. Li, J. Chen, Y. Li, T. Chen, J. Zou, and H. Wang, "Effect of polysaccharide of dendrobium candidum on proliferation and apoptosis of human corneal epithelial cells in high glucose," Medicine, vol. 96, no. 32, Article ID e7773, 2017.

[14] Z. Guo, Y. Zhou, J. Yang, and X. Shao, "Dendrobium candidum extract inhibits proliferation and induces apoptosis of liver cancer cells by inactivating Wnt/ $\beta$-catenin signaling pathway," Biomedicine \& Pharmacotherapy, vol. 110, pp. 371-379, 2019.

[15] H. Li, "Preventive effect and mechanism of ultrafine powder of Dendrobium candidum on photoaging model mice," Zhongguo Zhong Yao Za Zhi, vol. 44, pp. 4677-4684, 2019.

[16] Y. Shang, L. Zhao, W. Xi, G. Wen, and Z. Gao, "Primary culture and identification of adult dermal fibroblasts," $X i$ Bao Yu Fen Zi Mian Yi Xue Za Zhi, vol. 34, no. 9, pp. 824-828, 2018.

[17] P. Kumar, A. Nagarajan, and P. D. Uchil, "Analysis of cell viability by the MTT assay," Cold Spring Harbor Protocols, vol. 2018, no. 6, 2018 .

[18] S. D’Alessandro, Y. Corbett, S. Parapini et al., "Malaria pigment accelerates MTT-formazan exocytosis in human endothelial cells," Parasitology, vol. 146, no. 3, pp. 399-406, 2019.

[19] H. Rhee, M. Han, S. S. Kim et al., "The expression of two isoforms of matrix metalloproteinase- 2 in aged mouse models of diabetes mellitus and chronic kidney disease," Kidney Research and Clinical Practice, vol. 37, no. 3, pp. 222-229, 2018.

[20] A. M. Wojtowicz, S. Oliveira, M. W. Carlson, A. Zawadzka, C. F. Rousseau, and D. Baksh, "The importance of both fibroblasts and keratinocytes in a bilayered living cellular construct used in wound healing," Wound Repair and Regeneration, vol. 22, no. 2, pp. 246-255, 2014.

[21] A. Geiger, A. Walker, and E. Nissen, "Human fibrocyte-derived exosomes accelerate wound healing in genetically diabetic mice," Biochemical and Biophysical Research Communications, vol. 467, no. 2, pp. 303-309, 2015.

[22] A. Den Dekker, F. M. Davis, S. L. Kunkel, and K. A. Gallagher, "Targeting epigenetic mechanisms in diabetic wound healing," Translational Research, vol. 204, pp. 39-50, 2018.

[23] H. E. DesJardins-Park, D. S. Foster, and M. T. Longaker, "Fibroblasts and wound healing: an update," Regenerative Medicine, vol. 13, no. 5, pp. 491-495, 2018.

[24] P. J. Kallis and A. J. Friedman, "Collagen powder in wound healing," Journal of Drugs in Dermatology: JDD, vol. 17, no. 4, pp. 403-408, 2018.

[25] G. Li, W. Xing, M. Zhang et al., "Antifibrotic cardioprotection of berberine via downregulating myocardial IGF-1 receptorregulated MMP-2/MMP-9 expression in diabetic rats," American Journal of Physiology-Heart and Circulatory Physiology, vol. 315, no. 4, pp. H802-H813, 2018.

[26] F. K. Tombulturk, T. Soydas, E. Y. Sarac et al., "Regulation of MMP 2 and MMP 9 expressions modulated by AP-1 (c-jun) in wound healing: improving role of Lucilia sericata in diabetic rats," Acta Diabetologica, vol. 56, no. 2, pp. 177-186, 2019.

[27] H. Tang, T. Zhao, Y. Sheng, T. Zheng, L. Fu, and Y. Zhang, "Dendrobium officinale Kimura et Migo: a review on its ethnopharmacology, phytochemistry, pharmacology, and industrialization," Evidance Based Complementary and Alternative Medicine, vol. 2017, Article ID 7436259, 19 pages, 2017.

[28] J. A. Teixeira da Silva and T. B. Ng, "The medicinal and pharmaceutical importance of Dendrobium species," Applied Microbiology and Biotechnology, vol. 101, no. 6, pp. 2227-2239, 2017.

[29] C. Mo, D. Shetti, and K. Wei, "Erianin inhibits proliferation and induces apoptosis of HaCaT cells via ROS-mediated JNK/ c-Jun and AKT/mTOR signaling pathways," Molecules (Basel, Switzerland), vol. 24, 2019.

[30] Y. Gong, Y. Fan, L. Liu, D. Wu, Z. Chang, and Z. Wang, "Erianin induces a JNK/SAPK-dependent metabolic inhibition in human umbilical vein endothelial cells," In Vivo (Athens, Greece), vol. 18, no. 2, pp. 223-228, 2004.

[31] C.-Y. Gong, Z.-Y. Yu, B. Lu et al., "Ethanol extract of dendrobium chrysotoxum Lindl ameliorates diabetic retinopathy and its mechanism," Vascular Pharmacology, vol. 62, no. 3, pp. 134-142, 2014.

[32] R. Fleischmajer, K. Kuroda, R. Hazan et al., "Basement membrane alterations in psoriasis are accompanied by epidermal overexpression of MMP-2 and its inhibitor TIMP-2," Journal of Investigative Dermatology, vol. 115, no. 5, pp. 771-777, 2000.

[33] W. H. Lee, J. P. Warrington, W. E. Sonntag, and Y. W. Lee, "Irradiation alters MMP-2/TIMP-2 system and collagen type IV degradation in brain," International Journal of Radiation Oncology Biology Physics, vol. 82, no. 5, pp. 1559-1566, 2012.

[34] L. D. Kozaci, A. Guner, G. Oktay, and G. Guner, "Alterations in biochemical components of extracellular matrix in intervertebral disc herniation: role of MMP-2 and TIMP-2 in type II collagen loss," Cell Biochemistry and Function, vol. 24, pp. 431-436, 2006.

[35] F. M. Ho, S. H. Liu, W. W. Lin, and C. S. Liau, "Opposite effects of high glucose on MMP-2 and TIMP-2 in human endothelial cells," Journal of Cellular Biochemistry, vol. 101, no. 2, pp. 442-450, 2007.

[36] Z. Jin, C. Gu, F. Tian, Z. Jia, and J. Yang, "NDRG2 knockdown promotes fibrosis in renal tubular epithelial cells through TGF- $\beta 1 /$ Smad3 pathway," Cell and Tissue Research, vol. 369, no. 3, pp. 603-610, 2017.

[37] Y. Qu, L. Zhang, Z. Kang, W. Jiang, and C. Lv, "Ponatinib ameliorates pulmonary fibrosis by suppressing TGF- $\beta 1 /$ Smad3 pathway," Pulmonary Pharmacology \& Therapeutics, vol. 34, pp. 1-7, 2015.

[38] J. B. Trojanek, J. Michalkiewicz, R. Grzywa-Czuba et al., "Expression of matrix metalloproteinases and their tissue inhibitors in peripheral blood leukocytes and plasma of children with nonalcoholic fatty liver disease," Mediators Inflammation, vol. 2020, Article ID 8327945, 14 pages, 2020.

[39] C.-J. Li, L. Lv, H. Li, and D.-M. Yu, "Cardiac fibrosis and dysfunction in experimental diabetic cardiomyopathy are ameliorated by alpha-lipoic acid," Cardiovascular Diabetology, vol. 11, no. 1, p. 73, 2012.

[40] B. Dong, Q. T. Yu, H. Y. Dai et al., "Angiotensin-converting enzyme-2 overexpression improves left ventricular remodeling and function in a rat model of diabetic cardiomyopathy," Journal of the American College of Cardiology, vol. 59, no. 8, pp. 739-747, 2012.

[41] H. J. Vira, V. D. Pradhan, V. D. Umare et al., "Role of MMP-2 and its inhibitor TIMP-2 as biomarkers for susceptibility to systemic lupus erythematosus," Biomarkers in Medicine, vol. 14, no. 12, pp. 1109-1119, 2020.

[42] J. H. Park, J. M. Shin, H. W. Yang et al., "Cigarette smoke extract stimulates MMP-2 production in nasal fibroblasts via ROS/PI3K, Akt, and NF- $\kappa \mathrm{B}$ signaling pathways," Antioxidants (Basel), vol. 9, 2020. 